
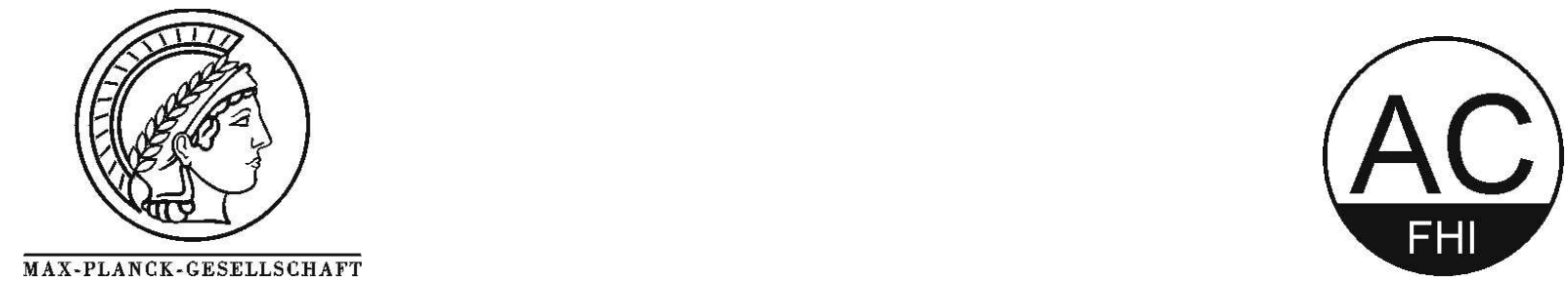

Review of Scientific Instruments, Vol. 77, 2006, 054102

\title{
A Molecular Beam Mass Spectrometer Equipped with a Catalytic Wall Reactor for In Situ Studies in High Temperature Catalysis Research
}

\footnotetext{
R. Horn, ${ }^{*}$ K. Ihmann, ${ }^{\dagger}$ J. Ihmann, F. C. Jentoft, M. Geske, A. Taha, K. Pelzer, and R. Schlögl

Department of Inorganic Chemistry, Fritz Haber Institute of the MPG, Faradayweg 4-6, 14195 Berlin, Germany.
} 


\section{Abstract:}

A newly developed apparatus combining a Molecular Beam Mass Spectrometer (MBMS) and a catalytic wall reactor is described. The setup has been developed for in situ studies of high temperature catalytic reactions $\left(>1000{ }^{\circ} \mathrm{C}\right)$, which involve besides surface reactions also gas phase reactions in their mechanism. The goal is to identify gas phase radicals by threshold ionization. A tubular reactor, made from the catalytic material, is positioned in a vacuum chamber. Expansion of the gas through a $100 \mu \mathrm{m}$ sampling orifice in the reactor wall into differentially pumped nozzle, skimmer and collimator chambers leads to formation of a molecular beam. A quadrupole MS with electron impact ion source designed for molecular beam inlet and threshold ionization measurements is used as analyzer. The sampling time from nozzle to detector is estimated to be less than $10 \mathrm{~ms}$. A detection time resolution of up to $20 \mathrm{~ms}$ can be reached. The temperature of the reactor is measured by pyrometry. Besides a detailed description of the setup components and the physical background of the method, this article presents measurements showing the performance of the apparatus. After deriving shape and width of the energy spread of the ionizing electrons from measurements on $\mathrm{N}_{2}$ and $\mathrm{He}$ we estimated the detection limit in threshold ionization measurements using binary mixtures of $C O$ in $N_{2}$ to be in the range of several hundred ppm. Mass spectra and threshold ionization measurements recorded during catalytic partial oxidation of methane at $1250{ }^{\circ} \mathrm{C}$ on a $\mathrm{Pt}$ catalyst are presented. The detection of $\mathrm{CH}_{3}$. radicals is successfully demonstrated.

Key words: Molecular Beam Mass Spectrometry, High Temperature Catalysis, Radicals, Threshold Ionization, Catalytic Wall Reactor, Methane Oxidation, Platinum 


\section{Introduction}

Molecular Beam Mass Spectrometry (MBMS) is a powerful tool to analyze reacting gas atmospheres for stable and short-lived species such as gas phase radicals. It has mainly been used in the past to investigate flames [1], plasmas [2] and chemical vapor depositions [3]. Although the molecular beam formation from a free jet source is a very complex process [4], the concept of the method is simple. A small portion of the reacting gases is expanded continuously through a tiny sampling orifice (nozzle) into a vacuum background. During expansion, collisions between the reacting atoms and molecules cease rapidly so that even reactive species like radicals are quenched. Up to two differentially pumped sampling cones (skimmer and collimator) downstream the nozzle cut out a molecular beam from the centerline of the expansion, which enters a mass spectrometer for analysis.

As MBMS images the composition of a reacting gas phase, applications to heterogeneous catalysis are few $[5,6]$. Heterogeneous catalytic reactions are commonly thought to occur on the surface of the catalyst, gas phase contributions are usually neglected. However, numerous industrially important reactions such as $H C N$ synthesis, ammonia oxidation or many hydrocarbon oxidations are performed at reaction temperatures beyond a thousand degrees Centigrade and at atmospheric or higher pressure. Under these conditions, surface and gas phase reactions may proceed in parallel and couple with each other by exchange of heat and reaction intermediates $[6,7,8]$. The apparatus described in this article has been developed to study such so called 'heterogeneous-homogeneous' reactions under high temperature and atmospheric pressure conditions. As many materials become catalytically active above a certain temperature, a sampling probe inserted into the reaction zone might interfere with the catalytic process under observation. For this reason we use a sampling orifice drilled directly into the catalytically active material that makes up the wall of the reactor. The reactor is placed in vacuum to enable free jet expansion through the orifice.

We begin our article with a brief discussion of relevant physical properties of free jet expansions and of the threshold ionization technique for the detection of radicals (Section 2). In Section 3 we provide a detailed description of the high temperature reactor, the vacuum system and the mass spectrometer. Section 4 presents finally test measurements to verify the capabilities of the apparatus.

\section{Physical Background}

\subsection{Molecular Beam Formation from Free Jet Sources}

Figure 1 shows schematically how the molecular beam is formed in our MBMS apparatus. The gases expand from the reactor tube (pressure $p_{0}$, density $\rho_{0}$, temperature $T_{0}$, velocity $v_{0}$ ) through a nozzle channel (diameter $d=100 \mu \mathrm{m}$, length $l=300 \mu \mathrm{m})$ into the vacuum background of the nozzle chamber 


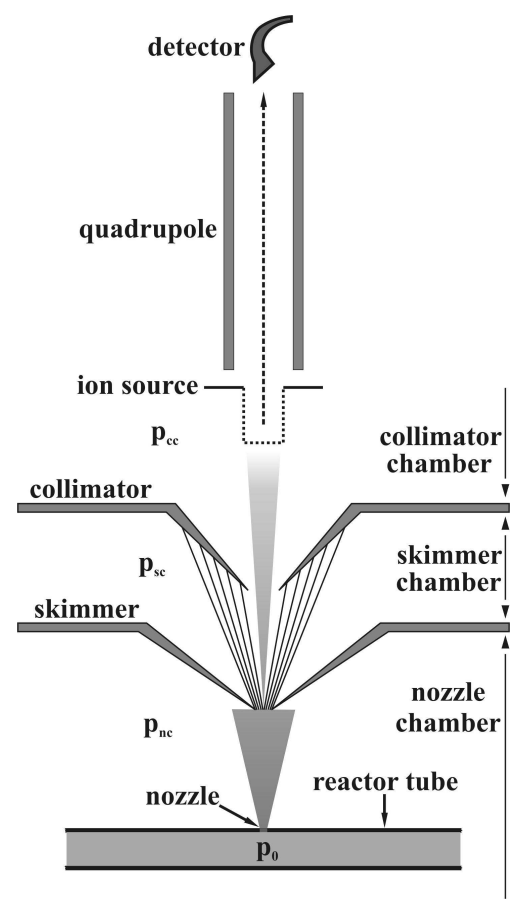

Figure 1: Molecular beam formation in the MBMS

(index $n c, p_{n c}<10^{-3}$ mbar). As the exiting molecules do not interact with surrounding walls behind the nozzle exit, the term 'free jet' expansion is appropriate. To understand the advantages and disadvantages of this sampling technique, a short outline of essential features of free jet expansions is presented hereafter.

\subsubsection{Shock Waves}

According to Euler's equation (1), the pressure gradient $p_{0} \rightarrow p_{n c}$ accelerates the molecules along the streamlines .

$$
-\left(\frac{d p}{\rho}\right)=v d v
$$

Integration of (1) from reactor conditions (index 0) to a point downstream in the expansion (index 1) gives:

$$
-\int_{p_{0}}^{p_{1}} \frac{d p}{\rho}=\frac{1}{2}\left(v_{1}^{2}-v_{0}^{2}\right)
$$

The integral on the left side of Equation (2) can be evaluated assuming a frictionless, adiabatic expansion (isentropic expansion, adiabatic exponent $\gamma=c_{p} / c_{v}, \gamma$ is the ratio of heat capacity at constant pressure and heat capacity at constant volume):

$$
\begin{array}{r}
\left(\frac{p}{p_{1}}\right)^{1 / \gamma} \cdot \rho_{1}=\rho \rightarrow \\
\int_{p_{0}}^{p_{1}} \frac{d p}{\rho}=\frac{1}{\gamma-1} \cdot \gamma \frac{p_{0}}{\rho_{0}} \cdot\left[\left(\frac{p_{1}}{p_{0}}\right)^{\frac{\gamma-1}{\gamma}}-1\right]
\end{array}
$$

Inserting (3) into (2) and substituting $\sqrt{\gamma p_{0} / \rho_{0}}=a_{0}(a=$ speed of sound $)$ gives:

$$
v_{1}^{2}-v_{0}^{2}+\frac{2}{\gamma-1} a_{0}^{2}\left(\left(\frac{p_{1}}{p_{0}}\right)^{\frac{\gamma-1}{\gamma}}-1\right)=0
$$

As the gas is at rest before expansion $\left(v_{0} \approx 0\right)$ and the speed of sound upstream the nozzle is related to the speed of sound in the expansion by $a_{0}^{2}=a_{1}^{2}\left(p_{0} / p_{1}\right)^{(\gamma-1) / \gamma}$, the Mach number of the molecules in the expansion $M a_{1}$ is given by:

$$
M a_{1}=\frac{v_{1}}{a_{1}}=\sqrt{\left(\frac{2}{\gamma-1}\right) \cdot\left[\left(\frac{p_{0}}{p_{1}}\right)^{\frac{\gamma-1}{\gamma}}-1\right]}
$$


Equation (5) is very important for the present application as it shows that if the pressure ratio $p_{0} / p_{1}$ is larger than $\left.((\gamma+1) / 2)\right)^{\gamma /(\gamma-1)}(\sim 2$ for most gases), the expansion becomes supersonic $\left(M a_{1}>1\right)$. In our MBMS the ratio between reactor pressure and pressure in the nozzle chamber is $\sim 10^{3} \mathrm{mbar} / 10^{-3} \mathrm{mbar}=$ $10^{6}$. For $N_{2},(\gamma=7 / 5)$ Eqn. 5 supplies a theoretical terminal Mach number of $M a_{1} \simeq 16$. Even though this value is not reached because transition to molecular flow prevents complete enthalpy conversion, it shows that the expansion is supersonic.

The consequence of the supersonic nature of the expansion is the formation of shock waves by which the expanding gas adapts to background conditions. Figure 2 shows the typical appearance of a free jet expansion. The shock waves forming radially around the

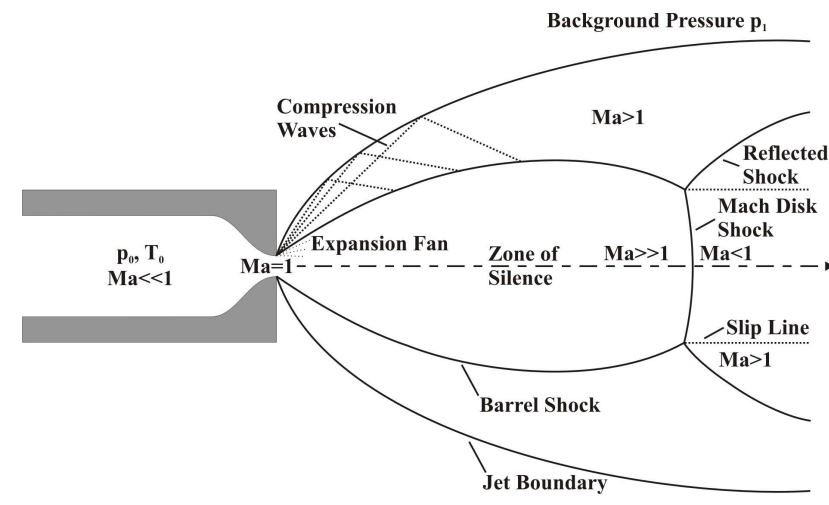

Figure 2: Continuum free jet expansion, adopted from [4, p. 15], changed

expansion are called barrel shocks. The shock wave perpendicular to the flow axis is the so called Mach disk. Shock waves are regions of high density gradients having thick- nesses of the order of the mean free path. As the molecules collide frequently within a shock wave, sampling of reactive species is optimally performed in the absence of any shock wave structure. The distance between Mach disk and nozzle exit $x_{m}$ in multiples of the nozzle diameter $x_{d}\left(x_{m} / d_{n}\right)$ can be estimated using an experimentally obtained relationship [9]:

$$
\frac{x_{m}}{d_{n}}=\frac{2}{3} \cdot \sqrt{\frac{p_{0}}{p_{n c}}}
$$

The pressure ratio $p_{0} / p_{n c}$ in our MBMS, which is in the order of $10^{6}$, leads therefore to a nozzle - Mach disk distance of several centimeters. As our skimmer is typically closer than $1 \mathrm{~cm}$ to the nozzle exit we extract our molecular beam from the zone of silence, well upstream the Mach disk (cp. Fig. 2). Furthermore, as the thickness of the shock waves at $p_{n c} \sim 10^{-3}$ mbar is in the range of several centimeters there is no distinct shock wave structure in the expansion at all.

\subsubsection{Quenching of Reactive Colli- sions}

Figure 3, taken from Miller's article on free jet sources in Atomic and Molecular Beam Methods (editor G. Scoles) [4], shows the behavior of velocity $v$, temperature $T$, particle density $n$ and binary hard sphere collision frequency $\nu$ along the centerline of the expansion. It is the steep drop in $T, n$ and $\nu$ that makes a free jet expansion the method of choice to freeze the chemical composition of rapidly reacting gases. The temperature of a monoatomic gas at a distance of 10 nozzle 


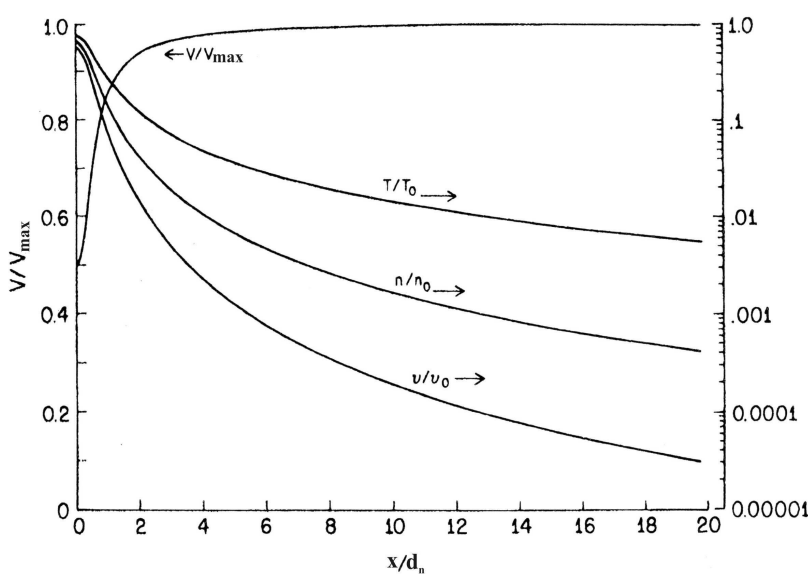

Figure 3: Free jet centerline properties versus distance $x$ in source diameters $d_{n}, \gamma=5 / 3$, temperature - $T$, particle density - $n$, binary hard sphere collision frequency $-\nu$, normalized by source stagnation values $T_{0}, n_{0}, \nu_{0}$, adopted from [4, p. 18], changed

diameters from the nozzle exit $(1 \mathrm{~mm}$ for our nozzle diameter of $0.1 \mathrm{~mm}$ ) is only about $1 \%$ of its value before expansion. As pointed out by Fenn [9], the flow velocities are a few times $10^{4} \mathrm{~cm} / \mathrm{s}$, and hence the cooling rate is of the order of $10^{8} \mathrm{~K} / \mathrm{s}$. Particle density and collision frequency decline even more steeply. At some point in the expansion the flow changes from continuum to free molecular flow (quitting surface model [4]).

Chemical reactions are mediated by intermolecular collisions. The quenching of collisions in the free jet expansion stops any chemical reactions. There are also no walls to which the molecules can stick and react. Free jet sampling provides therefore a snapshot of the chemical composition of the at- mosphere at the nozzle if the reactions to be studied proceed more slowly than the time needed to quench intermolecular collisions. The heterogeneous-homogeneous reactions that we are going to study with this setup, as e.g. hydrocarbon oxidations, proceed on a timescale of milliseconds [10]. The numerical values cited above show that chemical reactions will be quenched in the beam within a much shorter time span. Free jet expansion is therefore the appropriate way to sample gas phase species that are formed during these reactions.

\subsubsection{Mass Separation}

Another phenomenon occurring in molecular beam sampling from free jet expansions is mass separation. Mass separation is of special importance for quantitative measurements because it causes the composition of the gas mixture reaching the ionizer to be different from the composition at the place of sampling. Four mechanisms are discussed in the literature to explain the mass separation phenomenon:

i) pressure separation [11]

ii) free molecular diffusion [12]

iii) skimmer induced separation [13]

iv) background penetration [14]

i) Pressure separation occurs within a zone of a few nozzle diameters from the orifice where the pressure gradients are high and streamline curvature is severe. The lighter 
species are more strongly accelerated laterally so that heavier species are enriched in the beam.

ii) Molecular diffusion separation arises in the free molecular region of the beam. The thermal velocity spread of heavier particles perpendicular to the center line is less than that of lighter ones. This causes again an enrichment of heavier molecules in the central beam.

iii) Skimmer induced separation is caused by a shock wave rising up in front of the skimmer tip if it is placed in the continuum region. The inclined skimmer shock causes a mass separation by letting pass heavy molecules and deflecting lighter ones. This effect can be minimized by an elaborate skimmer design and by placing it downstream the transition to molecular flow.

iv) At progressed expansion the background pressure approaches that of the beam center. Light molecules have a higher diffusivity and can diffuse into the beam leading to their enrichment.

It is difficult to predict to which extent the various mechanisms contribute to mass separation in a given experiment. The general trend is that heavier species enrich in the center of the expansion, so mechanisms i) - iii) seem to prevail. To obtain quantitative results from an MBMS experiment, the mass spectrometer signals have to be corrected for mass separation. The relationship required for the correction has to be determined experimentally using calibrated gas mixtures. From the brief description of the mechanisms above it is obvious that the correction factors need to be obtained under conditions similar to those of the real experiment.

\subsubsection{Cluster Formation}

The last feature of free jet expansions that is of importance for the present application is cluster formation. As outlined in Section 2.1.2, the gases cool substantially during the expansion. Even though the low background pressure behind the nozzle prevents condensation on a macroscopic scale, cluster formation can readily happen. Gas expansions are actually used to prepare clusters for model studies in physics and chemistry [15]. However, for Molecular Beam Mass Spectrometry as analytical tool, cluster formation is unwanted. The clusters might produce signals having the same mass/charge ratio as species of interest. Figure 4 shows the formation of water clusters during MBMS measurements on the catalytic combustion of methane performed by the authors in a previously described MBMS setup [6]. In this system, the reactor gases were expanded through a water cooled nozzle located at the reactor outlet. The formation of clusters indicates that in the expansion, the partial pressure of water became higher than the vapor pressure at this temperature (supersaturation) and stable condensation nuclei were formed. In the setup presented here, cluster formation is not a problem because the gases are expanded through an orifice in the hot reactor wall. The vapor pressure depends exponentially on the temperature, therefore supersaturation does not occur. 


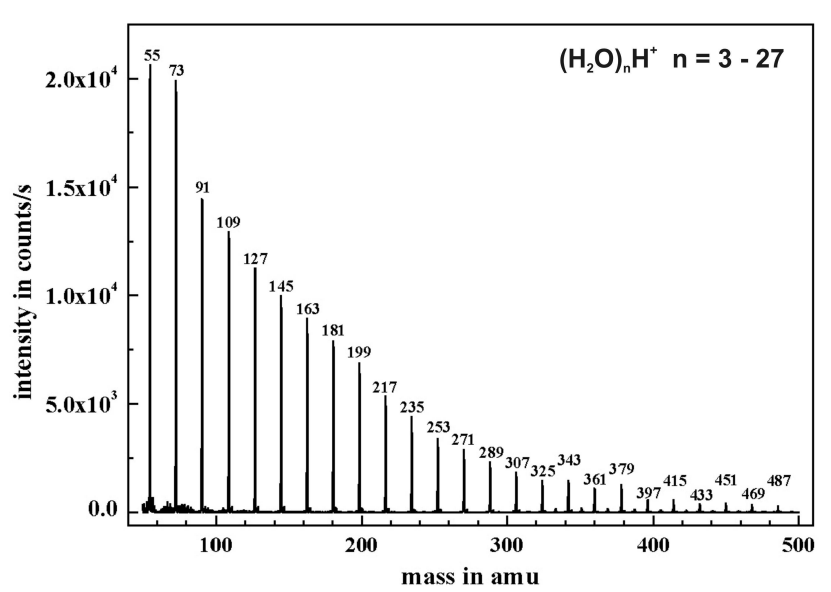

Figure 4: Formation of water clusters during free jet expansion of a gas mixture containing water vapor

\subsection{Radical Detection Threshold Ionization}

Mass spectrometry can be applied successfully for the detection of radicals [16]. The challenge in using this technique is to ionize the radicals selectively in the presence of interfering species. In the mass spectrometer described here, electron impact threshold ionization is used to achieve this goal. The principle of the method is as follows:

If an electron $\left(e^{-}\right)$possesses the energy $E$ and hits a molecule $(X Y)$, ionization and dissociation occur with certain probabilities $P_{i}$ and $P_{d}$ according to equations $(7)$ and (8) respectively:

$$
\begin{aligned}
X Y+e^{-} & \rightarrow X Y^{+}+2 e^{-} \\
P_{i}(E<I E(X Y)) & =0 \\
P_{i}(E \geq I E(X Y)) & =f_{i}(E)
\end{aligned}
$$

by

$$
\begin{aligned}
X Y+e^{-} \rightarrow X^{+}+Y+2 e^{-} & \\
P_{d}\left(E<A E\left(X^{+} / X Y\right)\right) & =0 \\
P_{d}\left(E \geq A E\left(X^{+} / X Y\right)\right) & =f_{d}(E)
\end{aligned}
$$

$I E$ means here the ionization energy of the molecule $X Y$ and $A E$ is the appearance energy of the fragment $X^{+}$from the molecule $X Y$. It can be shown by means of thermodynamics, that the ionization energy of the corresponding radical $X$. is always lower than the appearance energy of $X^{+}$from $X Y$ by an amount equal to the dissociation enthalpy $\left(\Delta_{d} H\right)$ of the $X-Y$ bond [16]:

$$
A E\left(X^{+} / X Y\right)=\Delta_{d} H(X-Y)+I E(X \cdot)
$$

To selectively detect the radical $X$. in presence of the molecule $X Y$, the ionization energy needs to be higher than the ionization energy of $X$. but lower than the appearance energy of $X^{+}$from $X Y$.

Bond dissociation enthalpies of single bonds like $\mathrm{RO}-\mathrm{H}, \mathrm{R}_{2} \mathrm{~N}-\mathrm{H}, \mathrm{R}_{3} \mathrm{C}-\mathrm{H}$ are in the order of $3-5 \mathrm{eV}$, therefore radicals like $\mathrm{OH} \cdot \mathrm{NH}_{2}$ or $\mathrm{CH}_{3}$. can be detected using conventional ionizers with electrons emitted from heated filaments. It is important that the energy spread of the ionizing electrons is smaller than $\Delta_{d} H(X-Y)$. One way to detect radicals by threshold ionization is to measure an Ionization Efficiency curve (IEcurve) around the threshold region. An IEcurve is a plot of the signal intensity at a fixed $m / z$ value vs. the energy of the ionizing electrons. 


\section{System Design}

\section{$3.1 \quad$ Reactor}

The catalytic wall reactor shown schematically in Figure 5 and in operation in Figure 6 was designed to study catalytic partial oxidations of hydrocarbons.

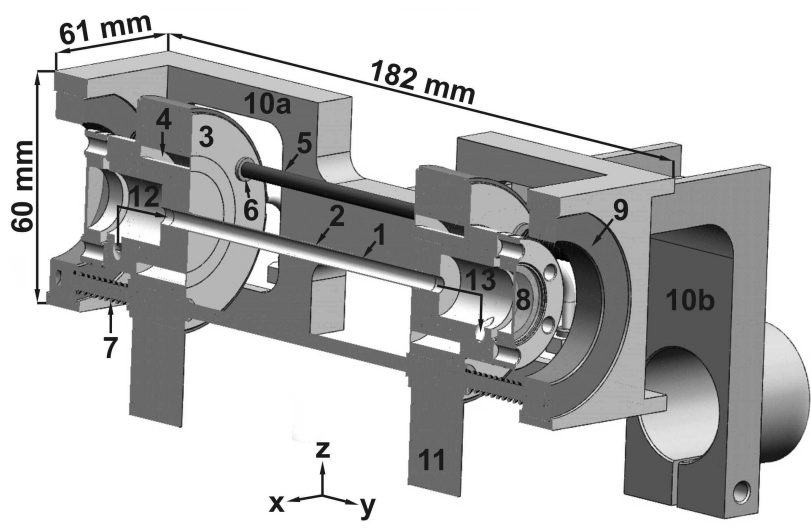

Figure 5: Catalytic wall reactor: 1) reaction tube, 2) nozzle position, 3) tube clamps, 4) water cooling, 5) mounting rods, 6) insulation bushings, 7) springs, 8) windows, 9) positioning rings, 10a; 10b) reactor holder, 11) electrical contacts, 12) gas in, 13) gas out

Catalytic wall reactors are well suited for high temperature reactions because heat and mass transfer between catalyst and gas phase is very efficient. The high volume to surface ratio of the reactor promotes gas phase chemistry and the cylindrical geometry is conveniently simple for simulation.

The reaction tube (length $=100 \mathrm{~mm}$, outer diameter $=5 \mathrm{~mm}$, inner diameter $=$ $4.4 \mathrm{~mm}$ ) is made of the catalytically active

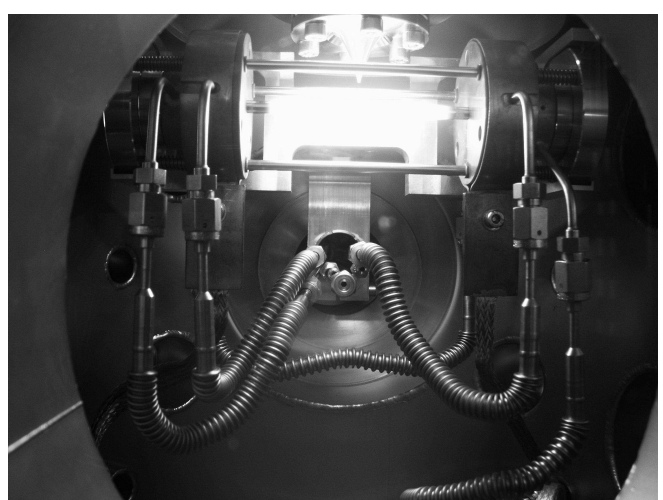

Figure 6: Catalytic wall reactor in operation, $T=1250{ }^{\circ} \mathrm{C}$

material, a $90 \% \mathrm{Pt} / 10 \% R h$ alloy. The nozzle which has a diameter of $100 \mu \mathrm{m}$ was fabricated by laser evaporation in the middle of the tube and aligned so that the molecular beam axis is in the $z$ direction (cp. coordinate systems in Fig. 5 and 8). A scanning electron micrograph of the nozzle is shown in Figure 7. The ends of the tube are shaped conically to fit tightly into cylindrical copper blocks. This way of fitting enables both, good electrical contact for heating and vacuum sealing. The copper cylinders have inlets and outlets for the reaction gases and cooling water as well as electrical contacts for the heating current. The water circulates through the annular spaces around the tube seats. Windows on both sides of the copper clamps enable optical access to the reaction tube. The reactor is stabilized by three stainless steel rods which pass in insulation bushings through the copper cylinders. The ends of the rods are clamped in aluminum rings which have a shape to form-fit into the reac- 


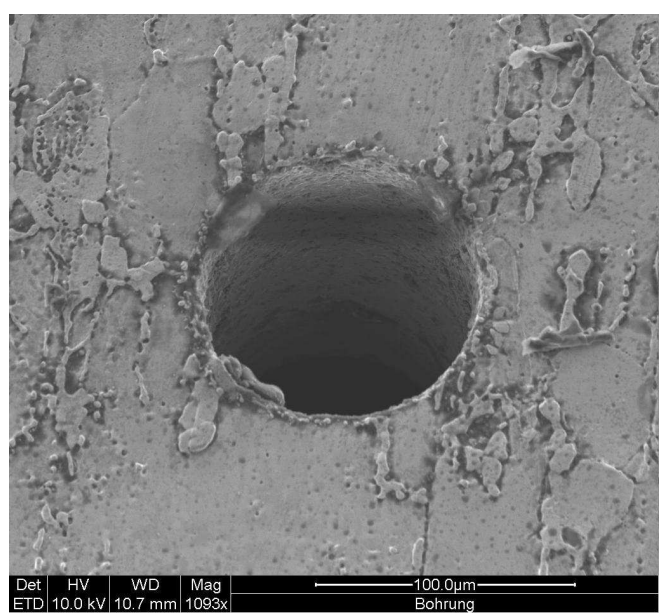

Figure 7: Scanning electron micrograph of the nozzle

tor holder. Springs between the copper cylinders and the end rings compress tube and fittings and compensate for thermal expansion if the reactor is heated. The reaction tube is heated by passing a DC current of up to $80 A$ through it. In the vacuum chamber heat is lost only by radiation and conduction axially through the tube and temperatures of up to $800{ }^{\circ} \mathrm{C}$ can be reached by electrical heating. The reactor can either be operated using the electrical heating or autothermally if the reaction is sufficiently exothermic. The reactor temperature is measured at the outer surface of the tube using a two wavelength pyrometer (type ISR 12-LO, Impac). The pyrometer works in quotient operation, i.e. the emission is measured at two wavelengths close to each other (1.52 and $1.64 \mu \mathrm{m})$ and the temperature is calculated from the quotient of these values. After calibration by comparison with a thermocouple, this prin- ciple makes the measurement independent of emissivity changes caused by changes of the tube surface. Also the curvature of the tube and the intervening window do not influence the temperature measurement.

\subsection{Vacuum System}

The core components of the MBMS system are shown in Figure 8 (simplified representation of the reactor). The nozzle cham-

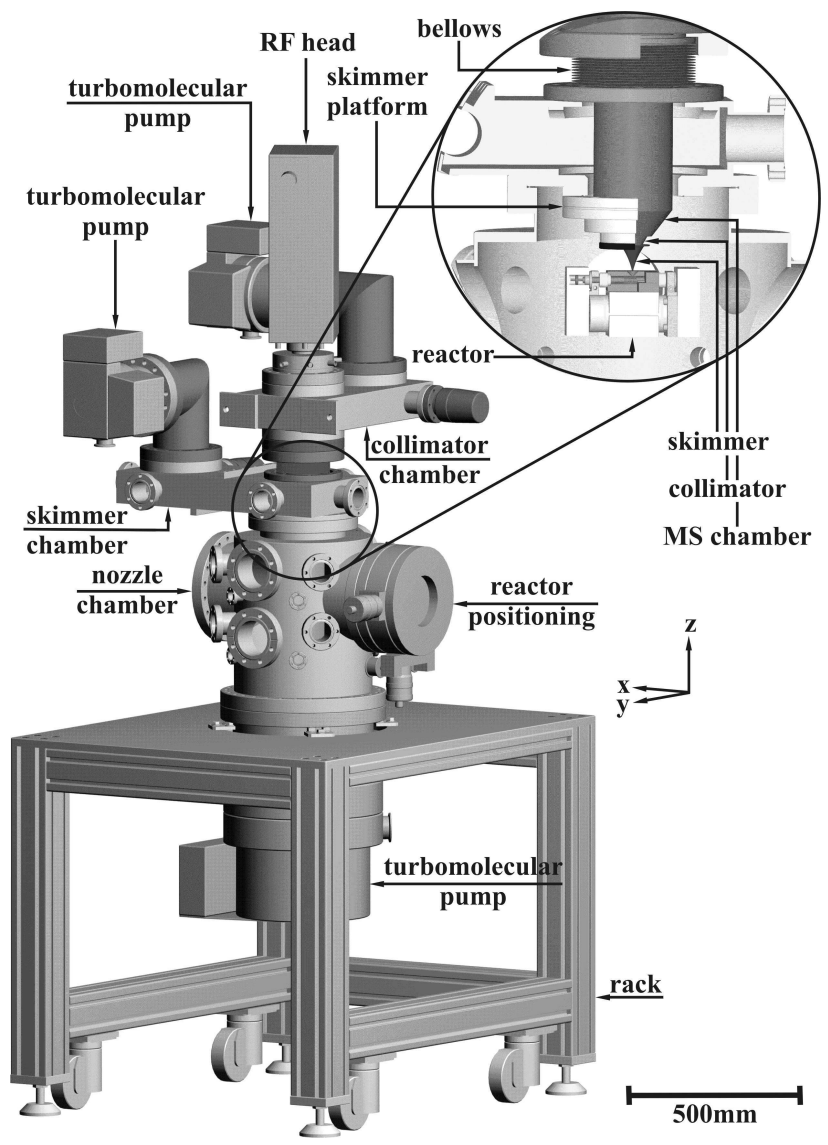

Figure 8: MBMS system - core components 
ber, which contains the reactor, is pumped by a high capacity turbomolecular pump (type TMU1601PCH, Pfeiffer Vacuum, $1400 l \cdot s^{-1} N_{2}$ ). Skimmer and collimator chamber are equipped with smaller turbomolecular pumps (type TMU261PCH each, $\sim 200 l \cdot s^{-1} N_{2}$ ). All turbomolecular pumps are backed by a combination of a roots blower and a membrane pump (RP35C Vario, Vacuubrand, $\left.\sim 35 \mathrm{~m}^{3} \cdot \mathrm{h}^{-1}\right)$. The pressure in the nozzle chamber is measured using a membrane capacitance diaphragm gauge (CMR275, Pfeiffer Vacuum, 0.11 $10^{-5}$ mbar, temperature controlled). Penning cold cathode gauges (type IKR261, Pfeiffer Vacuum) measure the pressure in the skimmer and collimator chamber respectively. Typical pressures under operating conditions are $10^{-5}<p_{n c}<10^{-3}$ mbar, $10^{-6}<p_{s c}<10^{-5}$ mbar and $10^{-8}<p_{c c}<$ $10^{-6}$ mbar. The skimmer cone is made from aluminium with an orifice diameter of $500 \mu \mathrm{m}$. The collimator cone, supplied by Hiden Analytical Ltd., is made from nickel and has an orifice of $900 \mu \mathrm{m}$. Skimmer and collimator are exactly aligned, their distance can be varied by movable bellows. To bring the nozzle in line with skimmer and collimator, the reactor can be positioned in the $x, y, z$ direction using a cross table (cp. Fig. 8, only $y, z$ shown). By varying the distance between nozzle and skimmer the acceptance angle of the skimmer can be varied. The closer nozzle and skimmer are, the higher is the beam intensity behind the skimmer but the lower becomes the pumping efficiency behind the nozzle. Another limit is imposed by the high temperature of the reactor tube. To avoid melting of the skimmer and to use the pumpage behind the nozzle the distance between nozzle and skimmer is adjusted typically to $10 \mathrm{~mm}$. The entire design is very compact with a distance between nozzle and ionizer of about $8 \mathrm{~cm}$.

\subsection{Mass Spectrometer}

The mass spectrometer located in the collimator chamber is a triple mass filter quadrupole instrument (type HAL4 EPIC Low Energy) manufactured by Hiden Analytical Limited. It allows for the analysis of neutrals, radicals, as well as positive and negative ions with a mass scan range from $0.4-510$ amu. The electron impact ion source is of cross beam type, designed to work with a molecular beam sample inlet. If the electron energy is varied as in threshold ionization experiments, the emission is kept constant. The ion source filaments are made of thoriated iridium, a material that has a low work function and makes the filaments resistant against the high thermal stress they suffer during operation at low electron energy values. A single channel electron multiplier is used as detector. It is mounted off-axis to avoid impact of light quanta and non ionized species. There are numerous publications in the literature where the Hiden spectrometer was applied successfully to detect radicals using the threshold ionization technique $[17,18,19]$. By monitoring a single mass only a time resolution of up to $20 \mathrm{~ms}$ is possible. 


\section{System Performance} and Test Measurements

\subsection{Determination of the Elec- tron Energy Spread Func- tion}

The electron impact source in the mass spectrometer supplies energetically inhomogeneous electrons. Potential causes of the energy spread are:

i) heated filament $\rightarrow$ thermal energy spread

ii) potential drop along the filament

iii) potential gradients inside the source

iv) filament contaminations

v) contact potential between filament and source chamber

Application of the threshold ionization technique for the detection of radicals requires to know the electron energy spread. In the following is described how it has been obtained for our instrument.

The total energy of an impacting electron $E$ can be written as the sum of the energy accumulated during acceleration in the electric field $V$ and all ill-defined contributions summarized in $U$ :

$$
E=V+U
$$

If $m(U)=m(E-V)$ is the energy distribution of all ionizing electrons for a certain voltage setting $V$, the ion current that is measured at a specific $m / z$ ratio is the convolution between the ionization (or dissociation) probability function $P_{i,(d)}(E)$ and the electron energy distribution (spread) function. $C$ is a proportionality constant:

$$
i(V)=C \cdot \int_{I E}^{\infty} m(E-V) P_{i,(d)}(E) d E
$$

For the simple case of single ionization, $P_{i}(E)$ is given by Wannier's threshold law [20]:

$$
P_{i}(E)=C \cdot(E-I E)^{1.127}
$$

As we demonstrated earlier [6], the method of Morrison [21] can be used to obtain the electron energy spread function from measured ionization efficiency curves. Morrison showed that for a strictly linear threshold law, e.g. $P_{i}(E)=C \cdot(E-I E)^{1}$, the second derivative of Equation 11 returns the electron energy spread function reversed with respect to the voltage axis:

$$
\begin{aligned}
\frac{d^{2} i(V)}{d V^{2}} & =-\left.C \cdot m(E-V)\right|_{I E} ^{\infty} \\
& =C \cdot m(I E-V)
\end{aligned}
$$

It was shown in [6] that the method can also be applied if the threshold law is not strictly linear. An exponent of 1.127 as in Wannier's law instead of 1.000 causes only a slight distortion of the high energy side of the second derivative. Width and maximum position of the distribution are only marginally influenced. Figure 9 shows the application of the method to the IE curves of $N_{2}$ and 

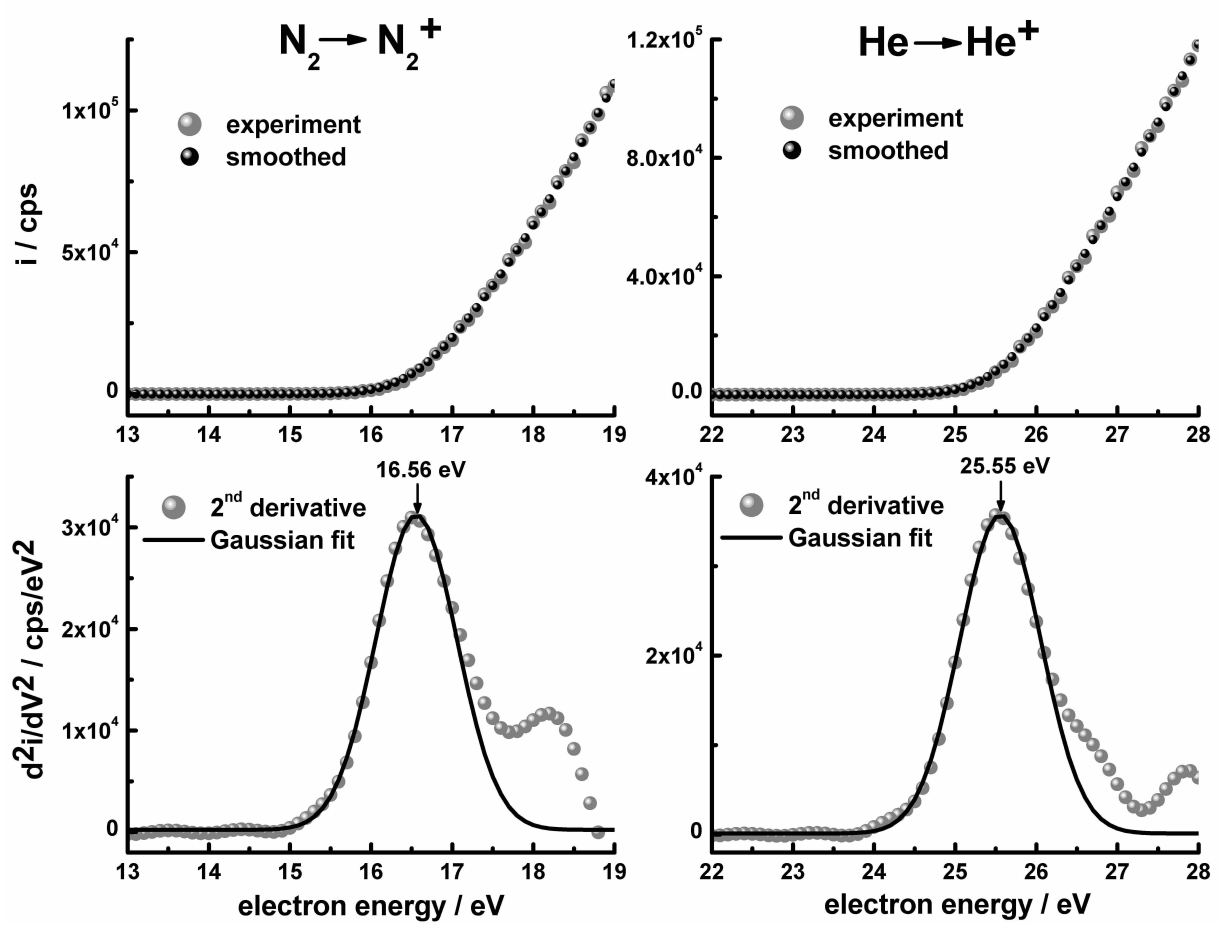

Figure 9: Determination of the shape of the electron energy spread function using the method of Morrison [21]

He (energy resolution $=0.1 \mathrm{eV})$. The data were smoothed before calculation of the second derivative (5 point FFT smoothing, Microcal Origin). The fits reveal that the energy spread can be described by a Gaussian distribution. For a Gaussian distribution, the maximum of the second derivative returns the ionization energy, here $I E_{H e, F i g .8}=$ $25.55 \mathrm{eV}, I E_{N_{2}, \text { Fig. } 8}=16.56 \mathrm{eV}$. Both values are about $1 \mathrm{eV}$ higher than the literature values $I E_{H e, l i t e r a t u r e}=24.56 \mathrm{eV}[22]$, $I E_{N_{2}}=15.48 \mathrm{eV}$ [23] which is just the energy offset of our instrument. The offset is caused by the work function of the filament material, making the displayed electron energy lower than the actual one. All energy values in Section 4.3 have been corrected for the offset. The deviation of the experimental values from the fit in Fig. 9 at higher energies might result from i) a nonlinear threshold law, ii) from overlapping ionization transitions or iii) from instrumental instabilities during the measurement process. Whereas instabilities might be minimized, nonlinearities and overlapping ionization transitions are inherent in the ionization process. $\mathrm{He}$ and $\mathrm{N}_{2}$ were chosen because they show a nearly linear threshold law without overlapping tran- 
sitions in the close threshold region. For $\mathrm{He}$ the next higher ionic state ${ }^{2} P_{1 / 2}$ is more than $40 \mathrm{eV}$ above the ground state ${ }^{2} S_{1 / 2}$. For $N_{2}$ the ionic ground state $X^{2} \Sigma_{g}^{+}$is at $15.48 \mathrm{eV}$, its next higher ionic states $A^{2} \Pi_{u}$ and $B^{2} \Sigma_{u}^{+}$ are at $16.60 \mathrm{eV}$ and $18.64 \mathrm{eV}$ respectively.

The shape of the spread function has been derived from Fig. 9. The width of the Gaussian distribution $\sigma$, the ionization energy $I E$ and the proportionality constant $C$ have been derived from a nonlinear fit of the discretized Equation 11 to the experimental data:

$$
\begin{array}{r}
i(V)=\frac{C}{\sigma \sqrt{2 \pi}} . \\
\sum_{I E / 0.1+1}^{n}\left[\operatorname{Exp}-\left(\frac{((j-1) \cdot 0.1-V)^{2}}{2 \sigma^{2}}\right) .\right. \\
\left.((j-1) \cdot 0.1-I E)^{1.127} \cdot 0.1\right]
\end{array}
$$

Figure 10 shows the results for $N_{2}$ and $H e$. The fit matches the experimental values closely, i.e Equations 11 and the discretized version 14 describe the curvature at the threshold very well. The ionization energies derived from the fit are $16.5 \mathrm{eV}$ for $\mathrm{N}_{2}$ and $25.5 \mathrm{eV}$ for $\mathrm{He}$ which, after substraction of the $1 \mathrm{eV}$ energy offset, are in excellent agreement with the literature values (15.48 eV [23] and $24.56 \mathrm{eV}$ [22] respectively). The energy spread varies only slightly over the energy range of nearly $10 \mathrm{eV}$ $\left(\sigma_{N_{2}}=0.49 \mathrm{eV}, \sigma_{\mathrm{He}}=0.47 \mathrm{eV}\right)$. If the energy spread can be considered constant, a simple criterion can be formulated for successful application of the threshold ionization technique: Any species of interest will be detected selectively if its ionization energy is

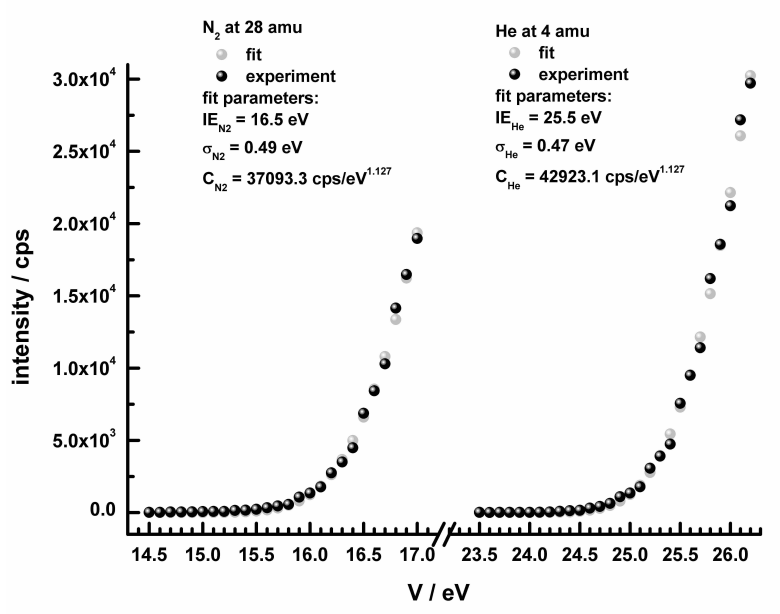

Figure 10: Fit of Eqn. 14 to experimental threshold data for $\mathrm{N}_{2}$ and $\mathrm{He}$ ionization, determination of width and offset of the electron energy spread function

separated by at least $2 \sigma \approx 1 \mathrm{eV}$ from the threshold energies of the interfering species.

\subsection{Detection Limits for Threshold Ionization Mea- surements}

To demonstrate the potential of the threshold ionization technique, the detection limit for the determination of $\mathrm{CO}$ in $\mathrm{N}_{2}$ has been determined. Both molecular ions ${ }^{12} \mathrm{C}^{16} \mathrm{O}^{+}$and ${ }^{14} N_{2}^{+}$overlay on $\mathrm{m} / z=28 \mathrm{amu}$ and $I E_{N_{2}}=$ $15.48 \mathrm{eV}$ [23] is only $1.41 \mathrm{eV}$ above $I E_{C O}=$ $14.07 \mathrm{eV}$ [24]. Seven calibration mixtures spanning a range from $0-20000$ ppm CO have been prepared and measured. Their actual $C O$ concentrations were determined using a non-dispersive infrared analyzer (type 
NGA2000, Rosemount). To obtain maximum specificity, the electron energy was set to $14.1 \mathrm{eV}$, i.e. only slightly above $I E_{C O}$. For this setting only the high energy tail of the electron distribution contributes to ionization. Additionally, the ionization cross section is very small so close to the threshold and the signal intensities are low.

The calibration data, the calibration line and the confidence interval are shown in Fig. 11. The intercept of the calibration

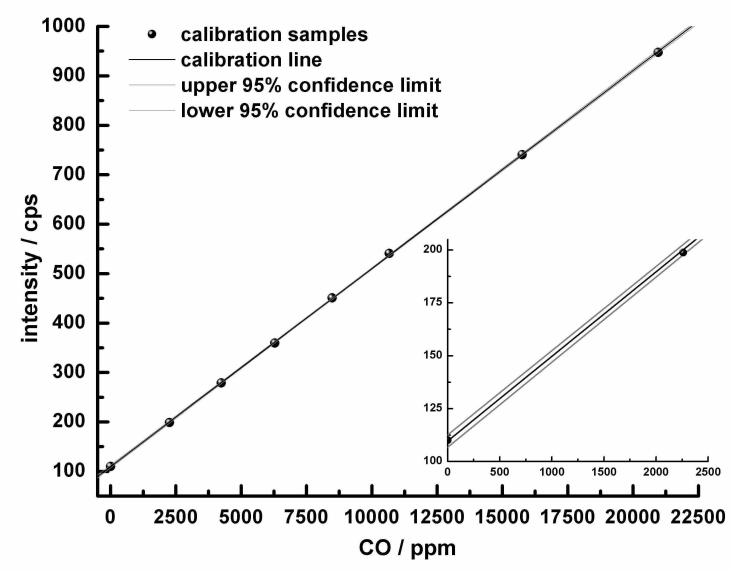

Figure 11: Calibration to determine the detection limit of $\mathrm{CO}$ in $\mathrm{N}_{2}$ using threshold ionization

line shows that there is only a small contribution of $\mathrm{N}_{2}$ ionization. The confidence interval is extremely narrow indicating high precision and linearity. The detection limit $(\alpha=\beta=0.05)$ for this model experiment, calculated from the calibration line according to [25], is $230 \mathrm{ppm}$. The detection limit in a radical detection experiment is certainly lower than the value obtained here, because the difference between the ionization energy of the radical and the appearance energy of the fragment is typically larger than $1.41 \mathrm{eV}$.

\subsection{Application Example - Radical Detection in Methane Catalytic Par- tial Oxidation}

To verify that the apparatus will sample and detect highly reactive gas phase species, the catalytic oxidation of methane has been chosen because i) numerical simulations predict gas phase radicals [26] and ii) because the mass spectra of the involved components are relatively simple with only few spectral interferences. Threshold ionization measurements were performed to search for gas phase radicals.

The reaction can be summarized by parallel occurrence of partial $\left(\mathrm{CH}_{4}+\frac{1}{2} \mathrm{O}_{2} \rightarrow\right.$ $\mathrm{CO}+2 \mathrm{H}_{2} \Delta_{r} \mathrm{H}^{\circ}=-36 \mathrm{~kJ} / \mathrm{mol}$ ) and total methane oxidation $\left(\mathrm{CH}_{4}+2 \mathrm{O}_{2} \rightarrow \mathrm{CO}_{2}+\right.$ $\left.2 \mathrm{H}_{2} \mathrm{O} \Delta_{r} H^{\circ}=-890 \mathrm{~kJ} / \mathrm{mol}\right)$. The overall reaction is highly exothermic and enables autothermal reactor operation. Numerical simulations predict the occurrence of $\mathrm{CH}_{3}$. and $O H$. in the gas phase but in concentrations close to the detection limit of the method (low ppm range). The radical concentration increases with temperature. A good indicator for gas phase chemistry is the formation of $C_{2}$ hydrocarbons. Numerical simulations show that the contribution of gas phase chemistry at atmospheric pressure is insignificant but becomes important at pressures of several bar $[26]$. 
To reach a high operating temperature, a mixture of $600 \mathrm{ml} / \mathrm{min} \mathrm{CH}_{4}, 500 \mathrm{ml} / \mathrm{min} \mathrm{O}_{2}$ $(C / O=0.6)$ and $200 \mathrm{ml} / \mathrm{min} \mathrm{He}$ (standard for energy axis) was used. In addition, $80 A$ were passed through the tube leading to a reactor temperature of $1250{ }^{\circ} \mathrm{C}$ (Fig. 6). The reactor pressure was slightly above atmospheric (1070 mbar), but because of the increased gas viscosity at high temperatures, the orifice flow was small and the pressure in the nozzle chamber was $<10^{-5}$ mbar. The pressure in the mass spectrometer chamber was $1.2 \cdot 10^{-7}$ mbar.

Fig. 12 compares the mass spectra and Fig. 13 the IE-curves at 15 amu for the reactor after light-off ('reaction on') and for the reactant mixture only ('reaction off').

Even though numerical simulations pre-

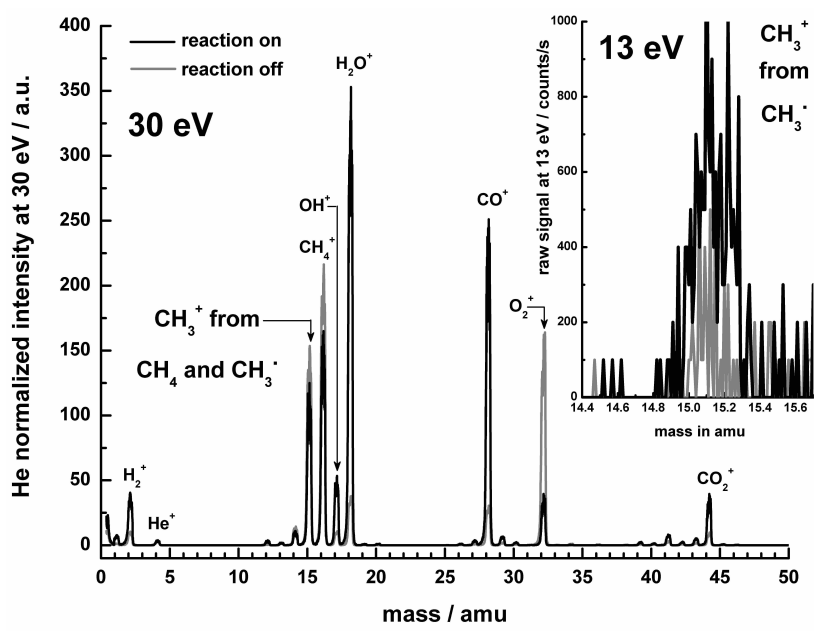

Figure 12: Mass spectra at $30 \mathrm{eV}$. Inset: $\mathrm{CH}_{3} \cdot$ signals at $13 \mathrm{eV}$

dict $O H$. in significant amounts besides $\mathrm{CH}_{3} \cdot \mathrm{OH}$. radicals could not be detected at 17 amu because at the ionization thresh-

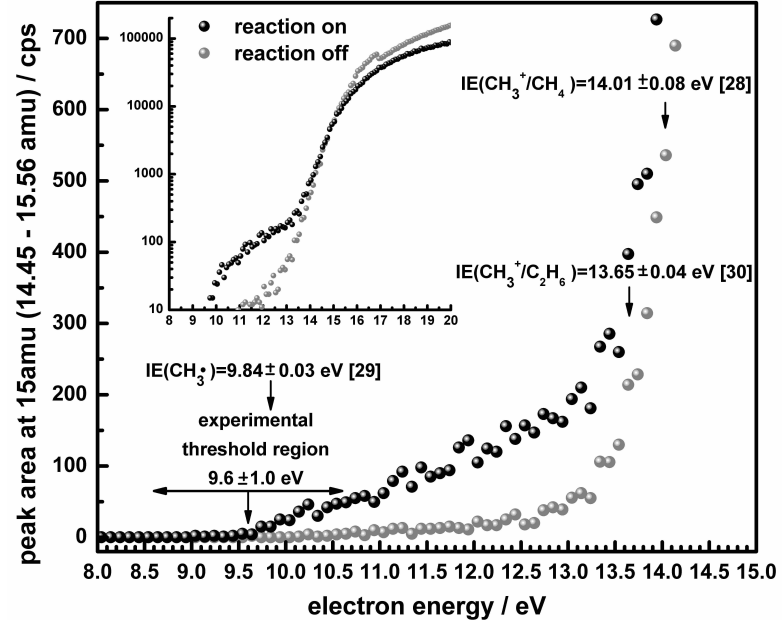

Figure 13: Detection of $\mathrm{CH}_{3}$. radicals at 15 amu by threshold ionization

old $I E\left({ }^{16} \mathrm{O}^{1} \mathrm{H}^{+} /{ }^{16} \mathrm{O}^{1} \mathrm{H} \cdot\right)=13.18 \mathrm{eV}$ [27] the $O H$. signal is obscured by the ionization of methane molecules containing ${ }^{13} \mathrm{C}$ $\left(\mathrm{IE}\left({ }^{13} \mathrm{C}^{1} \mathrm{H}_{4}^{+} /{ }^{13} \mathrm{C}^{1} \mathrm{H}_{4}\right)=12.63 \mathrm{eV}[28]\right)$.

The detection of $\mathrm{CH}_{3}$. radicals at $15 \mathrm{amu}$ was successful. The ionization energy of the radical $I E\left({ }^{12} \mathrm{C}^{1} \mathrm{H}_{3}^{+} /{ }^{12} \mathrm{C}^{1} \mathrm{H}_{3} \cdot\right)=$ $9.84 \mathrm{eV}$ [29]) is more than $4 \mathrm{eV}$ lower than the appearance energy of the $\mathrm{CH}_{3}^{+}$fragment $A E\left({ }^{12} C^{1} H_{3}^{+} /{ }^{12} C^{1} H_{4}=14.01 \mathrm{eV}\right)$ [28]. At an energy of $13 \mathrm{eV}$ which is sufficient to ionize $\mathrm{CH}_{3}$. radicals but too low for fragmentation of $\mathrm{CH}_{4}^{+}$into $\mathrm{CH}_{3}^{+}$, there is a small peak at $15 \mathrm{amu}$ (inset in Fig. 12). As shown in the inset, some radicals are detected even for the reaction off. The reason is that reactive collisions are rare under UHV-conditions and radicals desorbing from the chamber walls can cause a small but measurable background signal even if there are no radicals in the molecular beam coming from the 
reactor (reaction off). The problem of the radical background signal is known also from other applications and a mechanical chopper can be used to discriminate between beam and background [17]. For our application with the extremely hot reactor, a chopper is difficult to install in the beam path. We minimize the background contribution by using three differentially pumped vacuum chambers. In addition a larger nozzle will be used in further studies.

The presence of $\mathrm{CH}_{3}$. radicals in the reaction becomes clearer by looking at the IE-curves at 15 amu comparing the states reaction on or off (Fig. 13). With the reaction on, the IE curve rises linearly from about $9.6 \mathrm{eV}$ which agrees well with the ionization energy of $\mathrm{CH}_{3} \cdot(9.84 \mathrm{eV})$ before it increases steeply at about $13.5 \mathrm{eV}$ caused by $\mathrm{CH}_{3}^{+}$ formation from fragmentation of $\mathrm{C}_{2} \mathrm{H}_{6}$ $\left(\mathrm{IE}\left(\mathrm{CH}_{3}^{+} / \mathrm{C}_{2} \mathrm{H}_{6}\right)=13.65 \mathrm{eV}\right)[30]$ and $\mathrm{CH}_{4}$ $\left(\mathrm{AE}\left(\mathrm{CH}_{3}^{+} / \mathrm{CH}_{4}=14.01 \mathrm{eV}\right)\right)$ [28]. Without the reaction, the IE-curve remains close to zero until the fragmentation processes occur. Hence, it could be demonstrated that with the novel apparatus highly reactive gas phase species, present in a catalytic wall reactor under flow conditions at high temperatures, can be sampled and identified.

\section{Acknowledgement}

R. Horn acknowledges funding through an Alexander von Humboldt Foundation Feodor Lynen Fellowship.

\section{References}

[1] R. M. Fristrom, Int. J. Mass Spectrom. 16, 15 (1975).

[2] S. Park, F. Liao, and J. M. Larson et al., Plasma Chem. Plasma P. 24, 353 (2004).

[3] W. L. Hsu and D. M. Tung, Rev. Sci. Instrum. 63, 4138 (1992).

[4] D. R. Miller, Chapter 2 in Atomic and Molecular Beam Methods - Vol. 1 (Ed.: Scoles G.) (1988).

[5] Y. Feng, J. Niiranen, and D. Gutman, J. Phys. Chem. 95, 6558 (1991).

[6] R. Horn, G. Mestl, and M. Thiede et al., Phys. Chem. Chem. Phys. 6, 4514 (2004).

[7] R. J. Olsen, W. R. Williams, and X. Song et al., Chem. Eng. Sci. 47, 2505 (1992).

[8] D. J. Driscoll, K. D. Campbell, and J. H. Lunsford, Adv. Catal. 35, 139 (1987).

[9] J. B. Fenn, Int. J. Mass Spectrom. 200, 459 (2000).

[10] L. D. Schmidt, Stud. Surf. Sci. Catal. 130A, 61 (2000).

[11] F. S. Sherman, Phys. Fluids 8, 773 (1965).

[12] P. C. Waterman and S. A. Stern, J. Chem. Phys. 31, 405 (1959). 
[13] V. H. Reiss and J. B. Fenn, J. Chem. Phys. 39, 773 (1965).

[14] R. Campargue, J. Chem. Phys. 52, 1795 (1970).

[15] M. Kappes and S. Leutwyler, Chapter 15 in Atomic and Molecular Beam Methods - Vol. 1 (Ed.: Scoles G.) (1988).

[16] M. Sablier and T. Fujii, Chem. Rev. 102, 2855 (2002).

[17] H. Singh, J. W. Coburn, and D. B. Graves, J. Vac. Sci. Technol. A 17, 2447 (1999).

[18] C. A. Rego, R. S. Tsang, and P. W. May et al., J. Appl. Phys. 79, 7264 (1996).

[19] T. Schwarz-Selinger, V. Dose, and W. Jacob et al., J. Vac. Sci. Technol. A 19, 101 (2001).

[20] G. H. Wannier, Phys. Rev. 90, 817 (1953).

[21] J. D. Morrison, J. Chem. Phys. 21, 1767 (1953).

[22] R. C. Wetzel, F. A. Baiocchi, and T. R. Hayes et al., Phys. Rev. A 35, 559 (1987).

[23] K. Stephan, T. D. Märk, and J. H. Futrell et al., J. Chem. Phys. 80, 3185 (1984).

[24] E. Hille and T. D. Märk, J. Chem. Phys. 69, 4600 (1978).
[25] D. L. Massart, B. G. M. Vandeginste, and L. M. C. Buydens et al., Chapter 13 in Handbook of Chemometrics and Qualimetrics - Part A (1997).

[26] O. Deutschmann and L. D. Schmidt, AICHE J. 44, 2465 (1998).

[27] S. N. Foner and R. L. Hudson, J. Chem. Phys. 25, 602 (1956).

[28] P. Plessis, P. Marment, and R. Dutil, J. Phys. B 16, 1283 (1983).

[29] F. P. Lossing and G. P. Semeluk, Can. J. Chemistry 48, 955 (1970).

[30] P. Plessis and P. Marment, Can. J. Chemistry 65, 1424 (1987). 